\title{
Entre marchas, plebiscitos e iniciativas de ley: innovación en el repertorio de estrategias del movimiento No Más AFP en Chile (2014-2018)
}

Between demonstrations, plebiscites and law initiatives: innovation in repertoire of strategies of No Más AFP movement in Chile (2014-2018)

Joaquín Rozas Bugueño* - Antoine Maillet*

\begin{abstract}
Resumen: Las manifestaciones de No Más AFP emergieron en 2016, exigiendo el cambio al sistema de pensiones de Chile, utilizando un repertorio de estrategias variado. Este artículo tiene como objetivos describir el conjunto de tácticas usadas por el movimiento, más allá de la tradicional frontera insiders-outsiders; y explorar cuáles son los factores que determinan la innovación en el repertorio de estrategias del movimiento. Para ello, tomamos la propuesta de Rossi (2015), quien construye el concepto de repertorio de estrategias para superar la distinción entre repertorios convencionales y no convencionales. La innovación del repertorio de No Más AFP está asociada al contexto político - caso Myriam Olate, beneficios tributarios de las AFP y elecciones nacionales - y a definiciones internas - en particular la preocupación por la autonomía de los movimientos sociales respecto a los partidos políticos.
\end{abstract}

Palabras claves: Innovación del repertorio de estrategias; factores internos; factores externos; No Más AFP; Chile.

Abstract: No Más AFP demonstrations emerged in 2016, demanding the change of Chilean pension system, using a varied repertoire of strategies. This article aim at describing the set of tactics used by the movement, beyond the traditional distinction between insiders and outsiders; and at exploring the factors of innovation in repertoire of strategies. To do so, we build on Rossi (2015), who constructed the concept of repertoire of strategies to overcome the distinction between conventional and unconventional repertoires. The innovation in No Más AFP repertoire is the product of the political context - Myriam Olate case, AFP tax benefits and national elections - and internal definitions - especially the autonomy of social movements in relation to political parties.

Keywords: Innovation of repertoire of strategies; internal factors; external factors; No Más AFP; Chile.

Recibido: 30 julio 2018 Aceptado: 23 septiembre 2018

\footnotetext{
* Magíster en Sociología y Cientista Político, ambos grados de la Pontificia Universidad Católica de Chile. Correo electrónico: jirozas@uc.cl

${ }^{* *}$ Profesor Asistente del Instituto de Asuntos Públicos e Investigador Adjunto del Centro de Estudios de Conflicto y Cohesión Social (COES). Es Doctor en Ciencia Política de la Pontificia Universidad Católica de Chile y Sciences Po (Paris). Correo electrónico: antoinemaillet@iap.uchile.cl Ambos autores agradecen el apoyo del Fondecyt de Iniciación "Neoliberalismo híbrido" (CONICYT/FONDECYT/11150120) y del Centro de Estudios de Conflicto y Cohesión Social (CONICYT/FONDAP/15130009).
} 


\section{Introducción}

Previo al año 2011, Chile había sido catalogado por la literatura sobre movimientos sociales como un país políticamente estable y con bajos niveles de movilización social ${ }^{1}$, con la excepción del movimiento secundario de 2006 - la revolución pingüina ${ }^{2}$ - y protestas ambientales relativamente circunscritas a lo local ${ }^{3}$. Sin embargo, desde dicho año la situación cambió. Manifestaciones estudiantiles, medioambientales, territoriales, mapuche, feministas y en contra del sistema de pensiones se han tomado las calles chilenas ${ }^{4}$.

Autores como Roberts ${ }^{5}$ y Silva ${ }^{6}$ han señalado que los ciclos de movilización post 2011 en Chile tienen que ver con reclamaciones en contra de la desigualdad y el neoliberalismo. Esta entrada teórica es particularmente relevante en el caso de las movilizaciones en contra del sistema de pensiones chileno. Desde los años 80's, los países en desarrollo comenzaron a realizar cambios estructurales en materia social, recomendados por instituciones como el Banco Mundial y legitimados por el Consenso de Washington ${ }^{7}$.

Particularmente, Chile fue pionero en reformar la previsión y seguridad social, pasando de un sistema de reparto solidario a uno privado ${ }^{8}$. El sistema fue instaurado en dictadura a partir del Decreto de Ley n ${ }^{\circ} 3500$ de 1981, caracterizándose porque los afiliados aportan la totalidad del ahorro previsional - 10\% de sus ingresos - y son gestionados por las Administradoras de Fondos de Pensiones (AFP), organismos de carácter privado y con fines de lucro. Las AFP invierten los ahorros previsionales en el mercado de capitales tanto a nivel nacional e internacional con la finalidad de aumentar el ahorro de los cotizantes y generar utilidades para sí. Para el manejo de la inversión, el trabajador debe costear la gestión de las AFP a través de una comisión, equivalente entre $0,41 \%$ y $1,54 \%$ del salario, dependiendo de cada administradora 9 . Durante el primer gobierno Bachelet se elaboró una reforma que, si bien amplió ciertos beneficios, no modificó la estructura del sistema ${ }^{10}$. El segundo gobierno de Bachelet conformó una Comisión Asesora para revisar el sistema de pensiones y proponer una batería de iniciativas para reformar la capitalización individual.

En este contexto, el movimiento No Más AFP irrumpe en el escenario público con manifestaciones multitudinarias demandando un cambio estructural en el sistema de pensiones, en este caso, pasar del sistema de capitalización individual a uno de reparto tripartito y solidario. Desde aquel año, No Más AFP ha desarrollado un setvariado de acciones de protesta, desde acciones más tradicionales

\footnotetext{
${ }^{1}$ Sofía Donoso y Marisa von Bülow, Social Movements in Chile. Organization, trajectories \& political consequences, Nueva York, Palgraves Macmillan, 2017; Germán Bidegain, Autonomización de los movimientos sociales e intensificación de la protesta: estudiantes y mapuches en Chile (1990-2013), Tesis Doctoral en Ciencia Política, Pontificia Universidad Católica de Chile, 2015; Juan Pablo Luna y Fernando Filgueira, "The left turns as multiple paradigmatic crises", Third World Quarterly 30: 2, 2009, 371-395.

2 Ver: Sofía Donoso, "Dynamics of change in Chile: explaining the emergence of the 2006 pingüino movement", Journal of Latin American Studies 45: 1, 2013, 1-29.

3 Ver: Gonzalo Delamaza, Antoine Maillet y Christian Martínez, "Socio-territorial conflicts in Chile: configuration and politicization (2005-2014)", European Review of Latin American and Caribbean Studies 104, 2017, 23-46.

${ }^{4}$ Donoso y von Bülow, op. cit.; Víctor Muñoz y Carlos Durán, "Los jóvenes, la política y los movimientos en el Chile reciente. Ciclos sociopolíticos entre 1967 y 2017”, Izquierdas 45, febrero 2019, 129-159.

${ }^{5}$ Kenneth Roberts, “(Re)Politicizing Inequalities: Movements, Parties, and Social Citizenship in Chile”, Journal of Politics in Latin America 8: 3, 2016, 125-154.

${ }^{6}$ Eduardo Silva, "Post-Transition social movements in Chile in comparative perspective", en Sofía Donoso y Marisa von Bülow (compiladoras), Social Movements in Chile. Organization, trajectories \& political consequences, Nueva York, Palgraves Macmillan, 2017.

${ }^{7}$ Carmelo Mesa-Lago y Felipe Bertranou, "Pension reforms in Chile and social security principles, 1981-2015", International Social Security Review 69: 1, 2016, 25-45; Felipe Hevia de la Jara, "La difícil articulación entre políticas universales y programas focalizados. Etnografía institucional del programa Bolsa Familia de Brasil”, Gestión y Política Pública XX: 2, 2011, 331 -379.

8 Carmelo Mesa-Lago, "Reformas estructurales y re-reformas de pensiones, y sus efectos en el bienestar social: el caso de Chile", Politica y Sociedad 52: 3, 2015, 719-739.

${ }_{9}$ Comisión Asesora para el Sistema de Pensiones (2015).

10 Antoine Maillet, "Más allá del "modelo" chileno: una aproximación multi-sectorial a las relaciones Estado-mercado", Revista de Sociología e Politica, 23: 55, 2015, 53-73.
} 
como las marchas hasta la conformación de una propuesta técnica para el cambio en el sistema de AFP. En esta línea, este artículo busca responder las siguientes preguntas: ¿En qué ha consistido el repertorio de estrategias del movimiento No Más AFP? ¿Qué factores explican la capacidad innovadora de este movimiento y las decisiones de utilizar una acción en vez de otra?

La literatura tradicional sobre repertorios de movilización social y acciones de protesta se ha centrado en su carácter contencioso, lo que muestra una concepción de las acciones de los movimientos sociales como elementos que están al margen del sistema político ${ }^{11}$. En este artículo defendemos la idea de que la división entre lo institucional y lo extra-institucional o lo contencioso y lo rutinario es ficticia y que es más adecuado teóricamente referirse a repertorios de estrategias ${ }^{12}$, de tal forma de enmarcar las acciones de los movimientos sociales sin basarse en una distinción que no es operativa.

Además, para entender las opciones de los movimientos sociales en materia de acciones y tácticas de protesta, la literatura subraya dos factores, el ambiente político externo ${ }^{13}$ y las características internas de los movimientos sociales ${ }^{14}$. En este artículo, mostramos cómo condicionantes externas e internas de forma interrelacionada - elecciones nacionales, relación con actores políticos y las definiciones internas de la organización - son relevantes a la hora de analizar el repertorio de estrategias del movimiento No Más AFP. A su vez, un hallazgo interesante es que la autonomía política del movimiento en referencia a polity members permitió el desarrollo de un repertorio variado de acciones al no depender de los intereses y recursos de actores políticos, en la línea de los estudios de Somma y Medel ${ }^{15}$, Garretón ${ }^{16}$, Somma y Bargsted $^{17}$ y von Bülow y Bidegain ${ }^{18}$ que muestran que los movimientos sociales en Chile cada vez necesitan menos de los partidos políticos.

El caso de No Más AFP en Chile es relevante para la literatura de repertorios de movimientos sociales porque es un movimiento que innova y utiliza un amplio conjunto de acciones de protesta, lo que permite analizar en profundidad como operan las causas internas y externas en la elección de las

${ }^{11}$ Charles Tilly y Sidney Tarrow, Contentious politics, Nueva York, Oxford University Press, 2015, segunda edición; Sidney Tarrow, Power in Movement. Social Movements and Contencious Politics. Tercera Edición. Cambridge, Cambridge University Press, 2011, tercera edición; Charles Tilly, Regimes and Repertoires, Chicago, The University of Chicago Press, 2006; Doug McAdam, Sidney Tarrow y Charles Tilly, Dynamics of Contention, Cambridge, Cambridge University Press, 2001; Doug McAdam, Political Process and the Development of Black Insurgency, 1930-1970, Chicago, University of Chicago Press, 1999, segunda edición.

12 Donoso, op. cit.; Dan Wang y Sarah Soule, "Tactical innovation in social movements: the effects of peripherical and multiissue protest", American Sociological Review 81: 3, 2016, 517-548; Federico Rossi, "Conceptualizing strategy making in a historical and collective perspective”, en Federico Rossi y Marissa von Bülow (compiladores), Social movements dynamics. New perspectives on theory and research from Latin America, Londres y Nueva York, Routledge, 2015; Pettinicchio, David, "Institutional activism: reconsidering the insider/outsider dichotomy", Sociology Compass 6: 6, 2012, 499-510.

13 Tilly, op. cit., 2015; Tarrow, op. cit.; Tilly, op. cit., 2006; McAdam, op. cit.; Carlo Ruzza, "Institutional actors and Italian peace movement: specializing and branching out", Theory and Society 26, 1997, 87-127.

14 Wang, op. cit.; Rossi, op. cit.; Holly McCammon, The U.S. Women's Jury Movements and Strategic Adaptation: A More Just Verdict, Nueva York, Cambridge University Press, 2012; Craig Jenkins, "La Teoría de la Movilización de Recursos y el Estudio de los Movimientos Sociales", Zona Abierta 69, 1994, 5-49; Aldon Morris, "Black Southern Student Sit-In Movement: An Analysis of Internal Organization", American Sociological Review 46: 6, 1981, 744-67; John McCarthy y Meyer Zald, "Resource Mobilization and Social Movements: A Partial Theory", American Journal of Sociology 82: 6, 1997, 1212-1241. Para el caso chileno, ver por ejemplo Tokichen Tricot, "Movimiento de estudiantes en Chile: Repertorios de acción colectiva ¿algo nuevo?”, F@ro: Revista Teórica Del Departamento de Ciencias de La Comunicación, 15: 7, 2012.

15 Nicolás Somma y Rodrigo Medel, "Shifting relationships between social movements and institutional politics", en Sofía Donoso y Marisa von Bülow (compiladoras), Social Movements in Chile. Organization, trajectories \& political consequences, Nueva York, Palgraves Macmillan, 2017.

16 Manuel Antonio Garretón, La gran ruptura. Institucionalidad política y actores sociales en el Chile del Siglo XXI, Santiago, LOM ediciones, 2016.

17 Nicolás Somma y Matías Bargsted, "La autonomización de la protesta en Chile", en Juan Carlos Castillo y Cristián Cox (compiladores), Socialización politica y experiencia escolar: aportes para la formación ciudadana en Chile, Santiago, Editorial del Centro de Estudios de Políticas y Prácticas en Educación-Pontificia Universidad Católica de Chile, 2015.

18 Marisa von Bülow y Germán Bidegain, "It Takes Two to Tango: Students, Political Parties and Protest in Chile (20052013)" en Paul Almeida y Allen Cordero Ulate (compiladores), Handbook of Social Movements Across Latin America, Springer, 2015. 
acciones de protesta de los movimientos. Al mismo tiempo, la movilización en contra del sistema de pensiones es contingente y representa el fenómeno de la movilización en torno a la desigualdad que caracterizan a los ciclos de protesta en Chile post $2011^{19}$ donde, específicamente, no hay estudios sobre el movimiento No Más AFP.

La metodología utilizada en este artículo es cualitativa. Se triangulan datos a partir de revisión de prensa, revisión de documentos y literatura secundaria, y entrevistas en profundidad. En primer lugar, se analizaron 470 artículos de La Tercera online en un rango temporal de seis años - desde el 1 de enero de 2013 hasta el 22 de abril de $2018^{20}$. Los artículos fueron seleccionados bajo el criterio de si incluían o no las palabras "no más AFP" o "pensiones". La revisión de documentos consistió en el análisis de los programas presidenciales de Sebastián Piñera, Alejandro Guillier y Beatriz Sánchez; de información en la página de organizaciones que son parte de la Coordinadora No + AFP; y de artículos académicos que abordan el sistema chileno de pensiones. Finalmente, se realizaron 16 entrevistas en profundidad a actores relevantes del sector de pensiones en $\mathrm{Chile}^{21}$, entre los que destacan dirigentes del movimiento No Más AFP, altos cargos de gobierno, jefes programáticos de las principales campañas presidenciales de 2017 y expertos en seguridad social que colaboran con No Más AFP.

El artículo se estructura de la siguiente manera. Primero, se posiciona el estudio en la literatura de repertorios de movimientos sociales. Segundo, se describe el caso del movimiento No Más AFP. Tercero, se establece el análisis sobre el repertorio de estrategias de No Más AFP, los objetivos que persiguen cada una de las acciones y los factores internos y externos que permiten entender su conformación. Cuatro, se sintetizan los principales hallazgos a modo de conclusiones.

\section{Más allá de la distinción entre acción contenciosa y convencional: los repertorios de estrategias de los movimientos sociales}

Los repertorios que usan los movimientos sociales para posicionar y lograr sus demandas se han consagrado como una categoría analítica central del estudio de los movimientos sociales ${ }^{22}$. Son la agrupación de performances que despliegan los movimientos sociales con el fin de producir un cambio en su antagonista ${ }^{23}$. Lo contencioso de los repertorios tiene relación con el posicionamiento de reclamaciones o demandas de un movimiento social en confrontación a otro actor político relevante que es catalogado como responsable del malestar ${ }^{24}$.

Los estudios clásicos sobre política contenciosa han hecho una distinción entre actores que están dentro y fuera de la institucionalidad política y, con ello, estableciendo una diferencia entre acciones políticas insider-outsider ${ }^{25}$. No obstante, dicha distinción es poco útil analíticamente, puesto que la relación entre movimientos sociales e instituciones políticas es fluida, es decir, las acciones de un movimiento no

\footnotetext{
19 Silva, op. cit:; Roberts, op. cit.

20 También se utilizaron otras fuentes de prensa para completar la información.

21 Ver anexo.

22 Jennifer Beth Spiegel, "Performing 'in the red': transformation and tensions in repertoires of contention during the 2012 Quebec student strike. Social Movement Studies”, Journal of Social, Cultural and Political Protest 15: 5, 2016, 531-538; Tilly, op. cit., 2015; Tarrow, op. cit.; Tilly, op. cit., 2006.

23 Phillipe Hanna, Frank Vanclay, Esther Jean Langdon y Jos Arts, "Conceptualizing social protest and the significance of protest actions to large projects", The Extractive Industries and Society 3, 2016, 217-239; Spiegel, op. cit.; Patrick McCurdy, Anna Feigenbaum y Fabian Frenzel, "Protest camp and repertoires of contention", Journal of Social, Cultural and Political Protest 15: 1, 2015, 97-104; Takeshi Wada, "Modularity and transferability of repertoires of contention", Social Problems 59: 4, 2012, 544-571; Tilly, op. cit., 2006.

24 Tilly, op. cit., 2015; Tilly, op. cit., 2006; McAdam, op. cit., 2001.

25 Tarrow, op. cit;; Jenkins, op. cit.; Tilly, op. cit., 2006; McCarthy, op. cit.
} 
se posicionan estrictamente fuera de la institucionalidad, sino que depende de la decisión estratégica que éste adopte ${ }^{26}$.

En esta línea, distintos estudios han cuestionado la distinción entre acciones contenciosas y convencionales. Por un lado, Donoso 27 expone cómo en 2013 parte del movimiento estudiantil chileno se vuelca a la arena política institucional postulando a dirigentes universitarios al Parlamento, obteniendo cuatro diputaciones, difuminando la distinción entre política contenciosa y política rutinaria, asociada a movimientos sociales y a instituciones políticas formales respectivamente ${ }^{28}$. Por otro lado, Pettinicchio ${ }^{29}$ da cuenta de la poca utilidad de la rígida dicotomía entre actores institucionales y no institucionales en el estudio de los movimientos sociales, puesto que hay activistas que son parte de la institucionalidad política, que forman parte del Estado y que tienen un rol proactivo en la movilización social. En la misma línea, el autor sostiene que en ocasiones los actores outsiders pasan a ser insiders y viceversa.

En tanto, Rossi ${ }^{30}$ sostiene que teóricamente es más adecuado referirse a repertorios de estrategias en vez de repertorios contenciosos porque así no quedan fuera del marco de análisis aquellas acciones que escapan a lo que clásicamente se ha entendido por política contenciosa o no convencional - marchas, barricadas, tomas de recintos privados y/o públicos, mítines, entre otros. En este artículo adscribimos a esta crítica, por lo que nos centraremos en el concepto de repertorios de estrategias.

Con todo, los repertorios de estrategias desarrollados por movimientos sociales son una expresión más de la participación política, al igual que el voto o la participación en partidos políticos, siendo una práctica cada vez más común en las sociedades contemporáneas ${ }^{31}$, por lo que la distinción entre política convencional y no convencional es poco fructífera ${ }^{32}$. A continuación, se revisan factores externos e internos a los movimientos, que favorecen la innovación en las estrategias usadas por ellos.

\section{Factores externos de la innovación en los repertorios de estrategias}

Estudiar los repertorios es analizar las acciones de los movimientos sociales, que suponen una decisión estratégica en la interna de la organización ${ }^{33}$. Las estrategias están situadas cultural e históricamente, por lo que son dinámicas y variables ${ }^{34}$, "vary from place to place, time to time, and pair to pair" 35 . La literatura distingue entre condicionantes externos e internos para la innovación de los repertorios de estrategias ${ }^{36}$.

Por un lado, la idea de factores externos para la innovación en los repertorios de estrategia se refiere a que las acciones de los movimientos están imbricadas en los contextos políticos o ambiente

\footnotetext{
${ }^{26}$ Donoso, op. cit.; Rossi, op. cit:; Pettinicchio, op. cit.

${ }^{27}$ Sofía Donoso, “'Outsider' and 'insider' strategies: Chile's student movement, 1990-2014”, en Sofía Donoso y Marisa von Bülow (compiladoras), Social Movements in Chile. Organization, trajectories \& political consequences, Nueva York, Palgraves Macmillan, 2017.

28 Salieron electos como Diputados en las elecciones de 2013 Camila Vallejo (Partido Comunista), Giorgio Jackson (Revolución Democrática), Karol Cariola (Partido Comunista) y Gabriel Boric (Izquierda Autónoma).

${ }^{29}$ Pettinicchio, op. cit.

30 Rossi, op. cit.

31 Russell Dalton, Alix Van Sickle y Steven Weldon, "The individual-institutional nexus of protest behavior", British Journal of Political Science 40: 1, 2010, 51-73; Alan Schussman y Sarah Soule, "Process and protest: Accounting for individual protest participation", Social Forces 84: 2, 2005, 1083-1108; David Meyer y Debra Minkoff, "Conceptualizing Political Opportunity", Social Forces 82: 4, 2004, 1457-1493; Jack Goldstone, "More social movements or fewer? Beyond political opportunity structures to relational fields", Theory and Society 33, 2004, 333-365; Sidney Verba, Key Lehmann Schlozman y Henry Brady, Voice and Equality. Civic voluntarism in american politics, Cambridge, Massachusetts, Londres, Harvard University Press, 1995.

32 Donoso y von Bülow, op. cit.; Donoso, op. cit.; Rossi, op. cit.; Pettinicchio, op. cit.

33 Rodrigo Medel y Nicolás Somma, “'Marchas, ocupaciones o barricadas? Explorando las determinantes de las tácticas de la protesta en Chile”, Politica y gobierno XXIII: 1, 2016, 163-199; Rossi, op. cit.; Tilly, op. cit.

34 Hanna, op. cit.; Tilly, op. cit., 2015; Rossi, op. cit.

35 Tilly, op. cit., 2006, 35.

36 Idem; McCammon, op. cit.
} 
externo, lo que buena parte de la literatura ha llamado estructura de oportunidades políticas - EOP 37 . Tarrow $^{38}$ menciona que la EOP consta de cuatro dimensiones dinámicas y dos estables. Las dimensiones dinámicas son el nivel de acceso al sistema político, los cambios en los alineamientos políticos, la presencia o ausencia de aliados influyentes y la división de las élites, mientras que las dimensiones estables son la fortaleza de las instituciones públicas y la capacidad represiva del Estado.

La literatura ha señalado una relación entre repertorios y tipo de régimen político, teniendo distintos efectos el posicionamiento de una sociedad en el continuo autoritarismo-democracia. En este sentido, las condiciones para ampliar repertorios son más favorables en contextos democráticos que en autoritarios, ya que se permiten acciones contestatarias públicas y la capacidad represiva del Estado es más controlada ${ }^{39}$. Dependiendo del tipo de democracia y su contexto particular, existirán diferentes incentivos para la innovación de los repertorios.

Tilly y Tarrow ${ }^{40}$ señalan que la guerra fría sirvió como oportunidad al movimiento de los derechos civiles de Estados Unidos para innovar en su repertorio de acción, pasando de acciones legales a ocupaciones de lugares públicos, porque se veía políticamente incorrecto reprimir manifestaciones en contra de la segregación racial, mientras que los gobiernos estadounidenses desplegaban su política de "lucha por la libertad". Al mismo tiempo, Spiegel${ }^{41}$ muestra que el movimiento estudiantil de 2012 en Quebec pudo desarrollar un repertorio de estrategias amplio al reaccionar ante un gobierno que deslegitimaba sus acciones tradicionales de movilización como los paros universitarios, evidenciando la importancia de ir más allá del impacto que pueda tener o no el régimen democrático, sino cómo las acciones de las autoridades entregan oportunidades y restricciones a la conformación de repertorios por parte de los activistas.

Respecto a regímenes autoritarios, Scott ${ }^{42}$ muestra que las resistencias de los activistas en escenarios dictatoriales están caracterizadas por ser ocultas, detrás del anonimato y el disfraz político. No obstante, la disrupción de las acciones dependerá del contexto. Durante la primera mitad de los años 80's en Chile, bajo la dictadura de Pinochet, se realizaron jornada de protesta nacional multitudinarias, cuestión que en parte es explicada por la crisis económica internacional que afectó al país, tras la apertura económica por las reformas de la década del 7043.

Por otro lado, hay estudios que han vinculado a los aliados influyentes con los repertorios de estrategias. Investigaciones clásicas han argumentado que los movimientos sociales cercanos a actores políticos relevantes, como los partidos políticos, cuentan con mejores oportunidades para innovar en sus acciones de protesta ${ }^{44}$. Sin embargo, hay autores que han cuestionado dicha relación, especialmente en Chile ${ }^{45}$. Desde la vuelta a la democracia, la sociedad civil ha estado en un proceso de creciente distanciamiento con los partidos políticos, lo que se debería a la tesis de gobernabilidad de la Concertación, la que propugnaba la desmovilización de los actores sociales para evitar un retroceso autoritario $^{46}$. Según los estudios de Somma y Medel ${ }^{47}$ y Somma y Bargsted ${ }^{48}$, los movimientos sociales

37 Tarrow, op. cit.; Hanspeter Kriesi, "Political Context and Opportunity", en David Snow, Sarah A. Soule y Hanspeter Kriesi (compiladores), The Blackwell Companion to Social Movements, Oxford, Blackwell Publishing, 2006; Tilly, op. cit., 2006; David Meyer, "Protest and political opportunities", Annual Review of Sociology 30, 2004, 125-45; Meyer y Minkoff, op. cit.

38 Tarrow, op. cit.

39 Tilly y Tarrow, op. cit:; Tarrow, op. cit:; Tilly, op. cit.

40 Tilly y Tarrow, op. cit., 49-56.

${ }^{41}$ Spiegel, op. cit.

42 James Scott, Los dominados y el arte de la resistencia. Discursos ocultos, México D. F., Ediciones Era, 2000 [1990].

43 Cristopher Manzano, "La Asamblea de la Civilidad. Movilización social contra la dictadura en los 80", Santiago, Londres 38 , 2014.

44 Tilly y Tarrow, op. cit.; Tilly, op. cit.; Ruzza, op. cit.

45 Somma y Medel, op. cit.; Garretón, op. cit.; Somma y Bargsted, op. cit.; Bidegain, op. cit.

46 Silva, op. cit.; Garretón, op. cit.; Ruiz, Carlos. De nuevo la sociedad. Santiago, LOM Ediciones, 2015.

47 Somma y Medel, op. cit.

48 Somma y Bargsted, op. cit. 
cada vez necesitan menos a los partidos políticos para movilizarse, como también la relación con los movimientos sociales se ha vuelto prescindible para los partidos, en términos de conservar el poder.

Por su parte, Medel y Somma49 dan cuenta que los eventos de protesta con presencia de organizaciones políticas formales disminuyen las probabilidades que haya presencia de acciones disruptivas y violentas, lo que podría verse como una disminución de las chances de contar con innovaciones en el repertorio de estrategias de los movimientos. En este sentido, desde una teorización propia, aquí defendemos la idea de que la autonomía política de los movimientos sociales posibilita la innovación de los repertorios de estrategia porque no se depende de los recursos e intereses de actores de la elite del sistema político formal.

\section{Factores internos en la innovación de los repertorios de estrategias}

Por otro lado, los factores internos tienen que ver con la composición organizacional, las características de los activistas y el tipo de demanda de los movimientos sociales ${ }^{50}$. El nivel de estructuración interna de los movimientos sociales, la rutinización de sus acciones y la profesionalización de sus activistas como emprendedores de la movilización son fundamentales para determinar el tipo de estrategias que utilicen ${ }^{51}$.

Las características particulares en términos de composición de los movimientos sociales también son relevantes ${ }^{52}$. Schussman y Soule ${ }^{53}$ muestran que las personas que se identifican como liberales son personas que buscan mover el cerco de lo políticamente posible, como también Torcal, Rodón y Hierro ${ }^{54}$ argumentan que los individuos de izquierda tienen mayor predisposición a la movilización dado que las organizaciones de izquierda han desarrollado una tradición histórica respecto a la protesta como forma de participación política. En el caso de Chile, Garretón ${ }^{55}$ y Ruiz ${ }^{56}$ señalan que previo al golpe de Estado 1973, la izquierda chilena había desarrollado una trayectoria de movilización política al alero de sindicatos, el campesinado y los pobladores. A su vez, Medel y Somma ${ }^{57}$ señalan que en Chile los trabajadores utilizan repertorios disruptivos y no violentos - tomas de establecimientos, corte de calles y paros productivos, entre otros - por estar relacionados con la matriz económica productiva y que entre menor sea el número de convocados al evento de protesta más probable es que emerjan acciones disruptivas y violentas.

En cuanto a las reclamaciones que instalan los movimientos sociales, son relevantes tanto el tipo de demanda como también a qué actor va dirigida ${ }^{58}$. Wang y Soule ${ }^{59}$ argumentan que protestas multi-issue tienen más chances de desarrollar innovaciones porque se conjugan diferentes visiones e ideas desde grupos con diversas demandas. En Chile, Medel y Somma ${ }^{60}$ encontraron que las acciones que van dirigidas hacia el Estado suelen ser comunes - marchas y concentraciones - mientras que, cuando las reclamaciones están orientadas a empresas privadas, es más probable que los repertorios sean disruptivos y violentos.

\footnotetext{
${ }^{49}$ Medel, op. cit.

50 Wang, op. cit.; Rossi, op. cit.; McCammon, op. cit.; Jenkins, op. cit.; McCarthy, op. cit.

51 Jenkins, op. cit.; Morris, op. cit.; McCarthy, op. cit.

52 Mariano Torcal, Toni Rodon y María José Hierro, "Word on the street: the persistent of leftist-dominated protest in Europe", West European Politics 39: 2, 2016, 326-350; Alan Schussman y Sarah Soule, Sarah, "Process and protest: Accounting for individual protest participation”, Social Forces 84: 2, 2005, 1083-1108; Garretón, op. cit.; Ruiz, op. cit.

53 Schussman, op. cit.

54 Torcal, op. cit.

55 Garretón, op. cit.

${ }^{56}$ Ruiz, op. cit.

${ }^{57}$ Medel, op. cit.

58 Wang, op. cit.; Medel, op. cit.; David Meyer y Nancy Whittier, “Social Movement Spillover", Social Problems 41: 2, 1994, $277-98$.

59 Wang, op. cit.

${ }^{60}$ Medel, op. cit.
} 
Hecha la distinción entre condicionantes internas y externas de la innovación en los repertorios de estrategias, vale señalar que la relación entre ambos factores es dinámica y relacional, ya que las decisiones de los movimientos sociales pueden cambiar el entorno político, y con ellos las oportunidades y amenazas de la EOP, como también las características internas de los movimientos entregan la posibilidad de que éstos aprovechen o no las oportunidades del contexto ${ }^{61}$.

Con todo lo antes dicho, el caso del movimiento No Más AFP en Chile entrega una oportunidad para analizar en profundidad por qué los movimientos sociales hacen innovaciones en sus repertorios de estrategias. El movimiento en contra del sistema privado de pensiones, como se verá en próximas secciones, reúne variadas estrategias de protesta, desde marchas, pasando por reuniones con actores políticos, hasta propuestas técnicas para cambiar el sistema, permitiendo hacer un seguimiento de las causas del uso de las diferentes acciones, permitiendo entender la relación entre factores internos y externos de innovación en el contexto chileno.

Como se ha mostrado, la relación entre innovación en repertorios y factores externos e internos ha sido altamente estudiada por la literatura en general, sin embargo, para el caso chileno la situación es diferente, solo destaca el estudio de Medel y Somma ${ }^{62}$ - quiénes señalan que la táctica más común en Chile son las marchas y concentraciones, pero que existe un variado abanico de acciones - por lo que este estudio tiene la posibilidad de aportar en este tópico del estudio de los movimientos sociales en Chile. Para avanzar en este sentido, describimos los orígenes de No Más AFP.

\section{Movimiento No Más AFP: origen y organización}

\section{Origen de No Más AFP}

Si bien el movimiento en contra del sistema de capitalización individual emerge en la arena pública el año 2016, su formación data de mucho antes. Desde 2012, una serie de organizaciones de trabajadores y ciudadanos comenzaron a reunirse para discutir sobre la situación del sistema de capitalización individual y organizarse en pos de un cambio. En junio de 2013, en una reunión en la sede de la Asociación Nacional de Empleados Fiscales (ANEF), donde confluyeron organizaciones de Concepción, Rancagua y Santiago, nace la Coordinadora Nacional de Trabajadores No Más AFP con el único objetivo de terminar con el sistema de AFP, entendiendo que "(...) en el marco del actual sistema de capitalización [individual] no será posible mejorar las pensiones de nuestros actuales compatriotas. Lo que resulta más grave, de no efectuar cambios estructurales, es que este sistema nos condenará a pensiones mucho más precarias"63. En esta línea, los dirigentes de la Coordinadora definen a la organización como de "giro único", "[el] único objetivo es terminar con el modelo actual de las AFP e implementar un sistema basado en la lógica del reparto" 64.

Entre las organizaciones de trabajadores que fundaron la Coordinadora se encuentran la Confederación de Sindicatos Bancarios y Afines ${ }^{65}$, CONFUSAM, Federación Nacional de Profesionales Universitarios de los Servicios de Salud (FENPRUSS) y ANEF. Sin embargo, ANEF tiene una presencia "híbrida" en la Coordinadora, donde no cuentan con vocerías y no tienen participación formal, pese a que en dos Asambleas de la organización (2014 y 2017) acordaron participar de lleno en No Más AFP,

\footnotetext{
${ }^{61}$ Wang, op. cit.; McCammon, op. cit.; Goldstone, op. cit. ${ }^{62}$ Medel, op. cit.

${ }^{63}$ No Más AFP. Nuevo Sistema de Pensiones para Chile. Santiago, Coordinadora No Más AFP, $2016,8$.

${ }^{64}$ Entrevista $n^{\circ} 1$, Dirigente de No Más AFP, 16 de abril de 2018.

${ }^{65}$ De ahora en adelante Confederación Bancaria.
} 
situación que estaría dada por la conducción de partidos de la Nueva Mayoría en la ANEF, que no tiene interés particular en participar del movimiento ${ }^{66}$.

En el primer Congreso del movimiento en Valdivia (2014) se sumaron la Confederación Coordinadora de Sindicatos del Comercio ${ }^{67}$, la Unión Portuaria, la Federación de Asociaciones de Funcionarios Universidad de Chile (FENAFUCH) y organizaciones de trabajadores de Valdivia, Temuco, Osorno, Coyhaique, Antofagasta, Valparaíso y Arica ${ }^{68}$.

En abril de 2016, la Coordinadora celebró su segundo Congreso como organización en la Facultad de Medicina de Universidad de Chile. En aquella instancia acudieron más de 300 delegados de todo el país, los Diputados Gaspar Rivas ${ }^{69}$, Giorgio Jackson ${ }^{70}$ y Gabriel Boric ${ }^{71}$ y parte de los investigadores de la Fundación SOL. En dicha instancia se definieron las acciones para 2016,

(...) la tarea es implementar los zonales para fortalecer la estructura orgánica, impulsar el traspaso masivo de los afiliados de las AFP al fondo E; crear una Escuela de Formación Previsional; salir a las calles y plazas del país para informar a la gente y dar a conocer el proyecto de Seguridad Social con pensiones solidarias de un sistema de reparto que preparó en su momento la Coordinadora NO Más AFP, mientras, se sigue un trabajo de movilización que culmine en una protesta nacional y un paro nacional, usando la desobediencia civil pacífica masiva como herramienta para acabar con el sistema de $\mathrm{AFP}^{72}$.

Más adelante, se sumaría el Colegio de Profesores. La entrada de esta última organización fue posibilitada con la llegada de Mario Aguilar (Partido Humanista) a la presidencia, relegando al ex presidente Jaime Gajardo ${ }^{73}$ (Partido Comunista - PC) a la segunda vicepresidencia. "Lo que pasó fue que nosotros [Colegio de Profesores] nunca nos incorporamos oficialmente como organización, por qué, porque aquí en el gremio no era la línea de trabajo que tenía la directiva que gobernaba hasta enero del 2017 [PC]"74.

\section{Orgánica de No Más AFP}

Respecto a la organización interna de No Más AFP, lo primero que se debe mencionar es que el movimiento es una organización de hecho y no de derecho. La Coordinadora cuenta con una estructura de despliegue nacional, conformada por una dirección ejecutiva, un consejo nacional, un frente sindical y coordinaciones a nivel regional. La dirección ejecutiva es quien coordina las acciones a nivel nacional y está conformada por dos voceros de cada una de las cinco organizaciones pilares: CONFUSAM, FENPRUSS, Confederación Bancaria, Confederación del Comercio y Colegio de Profesores.

Por su parte, el consejo nacional es una instancia donde se reúnen la dirección ejecutiva y un representante de cada uno de los zonales - divididos en zonal norte, zonal centro norte, zonal centro sur y zonal sur $-\mathrm{y}$ un representante del frente sindical para definir la estrategia y acciones políticas.

En tanto, las coordinaciones regionales están conformadas por organizaciones de trabajadores y de ciudadanos, muchos de ellos con presencia estrictamente local. En dicha instancia, la Coordinadora

${ }^{66}$ Entrevista $\mathrm{n}^{\circ}$ 9, Dirigente de organización de trabajo que está fuera de No Más AFP, 16 de mayo de 2018.

${ }^{67}$ De ahora en adelante Confederación del Comercio.

${ }^{68}$ No Más AFP, op. cit.

${ }^{69}$ Diputado independiente y ex Renovación Nacional (RN) entre 2010 y 2018.

70 Diputado de Revolución Democrática, parte del Frente Amplio, desde el 2014 a la actualidad.

${ }^{71}$ Diputado de Movimiento Autonomista, parte del Frente Amplio, desde el 2014 a la actualidad.

72 Confederación Bancaria, "Tras exitoso $2^{\circ}$ Congreso Nacional Movimiento No + AFP toma fuerza", Confederación de Sindicatos Bancarios y Afines, 4 de mayo, 2016. Disponible en: http://bancariachile.cl/tras-exitoso-2o-congreso-nacional-movimientonoafp-toma-fuerza/. Revisado: 17 de mayo de 2018.

73 Gajardo estuvo a la cabeza del Colegio de Profesores desde 2007 hasta 2017.

${ }^{74}$ Entrevista n 5, Dirigente de No Más AFP, 8 de mayo de 2018. 
articula la expresión local de las acciones de despliegue nacional, como marchas y seminarios. Este nivel orgánico se creó en 2016 tras las marchas multitudinarias ${ }^{75}$.

Por último, tras el tercer congreso del movimiento, se creó el frente sindical. Este espacio nace como respuesta a la necesidad de que la Coordinadora pueda tener organizados a los sindicatos que no cuentan con presencia nacional y están circunscritos a sus territorios, para así poder desplegar un trabajo de concientización política con los trabajadores de dichos sindicatos.

Sí, lo que pasa es que el frente sindical se vuelve a retomar ahora en el congreso [de 2018 en Copiapó] como una definición de la posibilidad de seguir creciendo, siempre fue sindical esto y se irradió, y la verdad que en algunos territorios estas coordinadoras territoriales son muy sindicales, en otras son muy de pobladores y en otras son muy mezcladas, entonces qué es lo que nosotros quisimos en el congreso, resolver o empujar, con la formalización de este frente sindical, el tener un espacio más en la cultura de los sindicatos que no se perdiera (...) entonces, se plantean que varios sindicatos chiquititos que están muy puestos con la camiseta de ésta también tuvieran un espacio de reflexión y de avance en los temas ${ }^{76}$.

\section{Innovación en el repertorio de estrategias del Movimiento No Más AFP} (2014-2018)

El movimiento No + AFP tiene la particular característica de utilizar un repertorio de estrategias variado y novedoso. Entre las acciones de protesta se cuenta con marchas, llamados masivos a dejar ciertas AFP, cambiarse de fondos previsionales, elaboración de propuesta técnica de cambio al sistema de pensiones, plebiscito donde se preguntó si se quiere terminar o no con las AFP, reuniones con candidaturas presidenciales e iniciativa popular de ley. Estas acciones están asociadas a contextos políticos y definiciones internas. A continuación, analizamos estos repertorios en tres etapas. Vale destacar que se trata de un caso todavía abierto, donde podrían aparecer nuevas innovaciones a futuro.

\section{Repertorio de estrategias de No Más AFP antes y durante el gobierno de la Nueva Mayoría (2014-2018)}

La estrategia más común y menos novedosa ha sido la marcha. Las manifestaciones de la Coordinadora comenzaron desde su fundación, en 2013, pero con baja convocatoria y poco impacto mediático. El año 2016 sería el "decisivo" para la irrupción del movimiento. Uno de los factores que explica la gran convocatoria de la marcha de julio de 2018, fue el caso de Myriam Olate, ex Subdirectora Técnica de Gendarmería y ex esposa del Diputado Osvaldo Andrade ${ }^{77}$, quien se pensionó en 2015 por más de 5 millones de pesos en el sistema DIPRECA, relacionado directamente con el sueldo que recibió en los últimos seis meses, registrando un aumento en su última remuneración líquida de unos 2,5 millones de pesos aproximadamente ${ }^{78}$. En este sentido, "Eso [caso Olate] generó una indignación tal que la gente

\footnotetext{
75 Entrevista $n^{\circ}$ 5, op. cit.

${ }^{76}$ Entrevista ${ }^{\circ}$ 13, Dirigente de No Más AFP, 28 de junio de 2018.

77 Osvaldo Andrade se desempeñó como Diputado del Partido Socialista (PS) en los periodos 2010-2014 y 2014-2018. Fue Ministro del Trabajo y Previsión Social durante el primer gobierno de Michelle Bachelet (2006-2010).

78 Según Emol. "Ex subdirectora técnica de Gendarmería por pensión de \$5 millones: 'Es la que corresponde en derecho", Emol, 05 de julio, 2016. Disponible en: http://www.emol.com/noticias/Nacional/2016/07/05/810948/Myriam-Olatedefiende-pension-de-5-millones-Es-la-que-corresponde-en-derecho.html. Revisado: 14 de mayo de 2018, Olate registró un último sueldo líquido de 5.951 .549 de pesos, siendo que su sueldo anterior fue de 3.433 .495 pesos.
} 
consideró indispensable dar a conocer la molestia y la rabia en las calles"79. De esta manera, un gatillante de la masividad fue un factor político contextual, en la línea de la estructura de oportunidades políticas ${ }^{80}$.

Desde 2016 hasta hoy, la Coordinadora ha realizado 11 marchas, teniendo un peak de asistentes en la manifestación del 26 de marzo de 2017, logrando convocar a 800 mil personas en Santiago ${ }^{81}$. Además, el 4 de noviembre se convocó a un paro nacional donde participaron además de la Coordinadora, el movimiento Chile Mejor sin Tratado Transpacífico, la agrupación ciudadana "Aquí la Gente" y estudiantes secundarios. El Ministerio del Interior cifró la cantidad de manifestantes del paro en 75 mil personas a nivel nacional ${ }^{82}$.

Sobre el paro nacional, hay que subrayar que se ha posicionado dentro del imaginario colectivo de la Coordinadora como una acción de primera necesidad, considerada como la única con potencialidad real de hacer caer al sistema de AFP, cuestión que evidencia la relevancia de los factores internos, como el desarrollo de símbolos en la organización social, a la hora de analizar los repertorios de estrategias ${ }^{83}$.

No es suficiente en términos de la capacidad de demandar desde las calles con cientos de miles o millones de personas, creo que podemos hacer cinco, seis, diez marchas de millones de personas (...) todos tenemos la certeza, el convencimiento de la necesidad de una huelga general en el país para poder cambiar en definitiva el sistema, no hay otro camino, y una huelga general supone parar todo lo que es la producción de bienes y servicios en el país, parar toda la producción de bienes y servicios o sea son palabras mayúsculas ${ }^{84}$.

Por otro lado, el movimiento utilizó también como parte de su repertorio el llamado a las personas a cambiarse al Fondo E de las AFP 85 y a salirse de las AFP Cuprum y Provida. El primer llamado se dio en el contexto de la manifestación del 16 de octubre de 2016 y tenía por objetivo proteger a los afiliados del vaivén de la especulación financiera.

El trabajador no tiene por qué saber de mercados bursátiles y ni de inversiones (...) nosotros [No Más AFP] luchamos por seguridad social, eso es lo que le favorecería a la mayoría no a la minoría. La estrategia de refugiarse en el fondo E, es porque [las AFP] se focalizan en cuánto tú puedes ganar de rentabilidad, pero no te dicen que lo que puedes ganar en un mes, lo puedes perder todo y más al mes siguiente, entonces nosotros decíamos, preferible no ganar tanto y tener su fondo ahí seguro $^{86}$.

Por su parte, la segunda acción fue efectuada tras conocerse públicamente el beneficio tributario de Cuprum por su fusión con Argentum que ascendía a 130 millones de dólares, mientras que Provida hizo lo propio con Acquisition, beneficiándose tributariamente por 288 millones de dólares ${ }^{87}$. En un

${ }^{79}$ Esteban Maturana en La Tercera, "La historia del movimiento que convocó a miles de chilenos", 26 de julio, 2016a. Disponible en: http://www2.latercera.com/noticia/la-historia-del-movimiento-que-convoco-a-miles-de-chilenos/. Revisado: 14 de mayo de 2018.

80 Tarrow, op. cit.; Tilly, op. cit.; Kriesi, op. cit.; Meyer, op. cit.

${ }^{81}$ La Tercera, "Coordinadora "No + AFP" calificó como histórica la jornada de movilización", 26 de marzo, 2017a, Disponible en: http://www2.latercera.com/noticia/hoy-se-realiza-una-nueva-marcha-convocada-noafp/, Revisado: 14 de mayo de 2018.

82 La Tercera, "No + AFP cambia de estrategia y radicaliza presión al gobierno", 4 de noviembre, 2016b, Disponible en: http://www2.latercera.com/noticia/noafp-cambia-estrategia-radicaliza-presion-al-gobierno/, Revisado: 14 de mayo de 2018.

83 Wang, op. cit.; Rossi, op. cit.; McCammon, op. cit.; Meyer y Whittier, op. cit.

${ }^{84}$ Entrevista $n^{\circ} 1$, op. cit.

${ }^{85}$ Los fondos de inversión de las AFP se dividen en 5 (A, B, C, D y E), donde el fondo E es el de menor riesgo en términos de inversión.

86 Entrevista n 11, Dirigente de No Más AFP, 8 de junio de 2018.

${ }^{87}$ La Tercera, "SII recopila antecedentes por fusión entre AFP Provida y Acquisition", 3 de marzo, 2016c, disponible en: http://www2.latercera.com/noticia/sii-recopila-antecedentes-por-fusion-entre-afp-provida-y-acquisition/, revisado: 14 de mayo de 2018; La Tercera, "Director del SII califica como "legal” la fusión entre Cuprum y Argentum”, 6 de octubre, 2015, 
primer momento, las fusiones fueron aprobadas por la Superintendencia de Pensiones y el Servicio de Impuestos Internos, sin embargo, la Contraloría de la República impugnó la fusión de Cuprum y Argentum. Dicha situación significó la renuncia a Tamara Agnic, Superintendenta de Pensiones. En su lugar, asumió el cargo Osvaldo Macías.

Para la Coordinadora, la fuga de Cuprum y Provida tenía por objetivo hacer tambalear el sistema de AFP. En este sentido, “(...) si tú lograbas haber hecho el abandono en cantidades significativas los dejas sin el manejo económico y podrías haber hecho quebrar Provida y si quebrabas Provida habríamos dado la fuerza al movimiento para cambiar el sistema" 88 .

A fines de noviembre de 2016, la Coordinadora presentó su propuesta de cambio al sistema de pensiones. Esta opción propone un sistema de reparto tripartito y solidario administrado por un ente público, autónomo y sin fines de lucro. Los principios de la propuesta son solidaridad, universalidad, igualdad, suficiencia, sustentabilidad financiera, responsabilidad estatal en la gestión del sistema, participación de diferentes actores y eficiencia en los gastos administrativos ${ }^{89}$.

Este sistema de reparto se concibe como un mecanismo de solidaridad intergeneracional para la seguridad social, es decir, que los activos contribuyan a las pensiones de los pasivos, para que estos últimos reciban una jubilación acorde a sus años de cotización y remuneraciones. La propuesta se elaboró en base a supuestos conservadores para el periodo 2017-210090. Respecto al elemento tripartito, se propone un aumento gradual de 5,2\% ${ }^{91}$ en el total de la cotización hasta llegar al 18\% de cotización para 2024, donde el empleador aporte un 9\% del sueldo del trabajador y el trabajador aporte otro 9\%. El Estado contribuirá a través del presupuesto de la nación a las pensiones, aumentando de forma incremental el Fondo de Reserva Técnica (a explicar más adelante) hasta un 3\% del PIB para el año 2033, manteniéndose en ese gasto hasta el 210092. En tanto, se subsidian 5 años de pensiones a las mujeres por reconocimiento del trabajo doméstico y de cuidado no remunerado, además de sopesar la diferencia salarial por género ${ }^{93}$.

Una de las principales características de la propuesta es que cuenta con pensiones fijas a recibir de acuerdo con el ingreso promedio de los últimos 10 años de cotización en el nuevo sistema, estableciéndose una pensión máxima contributiva y una pensión mínima garantizada. El sistema considera también una pensión universal no contributiva - independiente de los años de cotización y densidad de contribución - equivalente al salario mínimo.

Al mismo tiempo, el sistema contará con un Fondo de Reserva Técnica el que estará conformado por el Fondo de Reserva de Pensiones y los excedentes de los gastos de gestión. Este Fondo será invertido en el mercado por parte de la entidad rectora del sistema para obtener utilidades. La idea es que este fondo pueda hacer cumplir las obligaciones del sistema en periodos de decrecimiento económico y previendo el impacto del envejecimiento de la población.

Para No Más AFP, la estrategia política detrás de la propuesta técnica era demostrar que el movimiento social puede elaborar propuestas de política pública con base técnica y sostenibilidad en el

disponible en: http://www2.latercera.com/noticia/director-del-sii-califica-como-legal-la-fusion-entre-cuprum-y-argentum/, revisado: 26 de mayo de 2018.

88 Entrevista $n^{\circ}$ 2, Dirigente de No Más AFP, 24 de abril de 2018.

${ }^{89}$ No Más AFP, op. cit.

90 Distribución de densidad de cotización del año 2015; crecimiento de 1\% del PIB anual; crecimiento de las remuneraciones imponibles reales de $1 \%$ al año; aumento de $1 \%$ anual del salario mínimo imponible; inflación promedio anual de 3,5\%; rentabilidad real anual del Fondo de Reservas Técnicas de Pensiones de 2,5\%; y tablas demográficas utilizadas por CEPAL.

91 Considerando que la cotización actual está dada por el 10\% del trabajador, el 1,38\% promedio que va a las comisiones de administración de las AFP y el 1,41\% dirigido al seguro de invalidez y sobrevivencia.

92 El Mercurio, “Esta es la propuesta de cambio al sistema de pensiones de No + AFP”, El Mercurio, 29 de noviembre, 2016, Disponible en: http://www.elmercurio.com/Inversiones/Noticias/Fondos-de-Pensiones/2016/11/29/Esta-es-la-propuestade-cambio-al-sistema-de-pensiones-de-NoAFP.aspx, Revisado: 28 de mayo de 2015.

${ }^{3}$ Se agregarán dos años extra de cotización cuando las mujeres cumplan 5 años de cotización y 3 años extra más cuando cumplan 10 años de cotización. 
tiempo, de forma de entregarle legitimidad a las demandas por terminar con el sistema de capitalización individual.

[La propuesta] tiene que ver con instalar al movimiento social con propuestas técnicas sólidas y eso en el fondo es decirle a la élite que no sólo ellos pueden pensar, sino que también hay otros que piensan maneras distintas de ver el país, básicamente es jugar en su cancha, que siempre ellos han utilizado a su haber, entrar a la cancha de ellos con propuestas sólidas y, por lo tanto, es validarnos como un movimiento social que tiene ideas, propuestas que no sólo quedan en el eslogan, ni en el reclamo, ni en la consigna, sino que van más allá y entregan una propuesta técnica totalmente sólida, absolutamente financiable y posible de lograr si es que hay voluntad política ${ }^{94}$.

En este sentido, la acción del movimiento fue exitosa, ya que desde el gobierno catalogaron la propuesta como técnicamente viable.

(...) Ellos [No Más AFP] tenían una propuesta que era bien contundente, nosotros la analizamos y les entregamos un resultado y ese resultado dice que su propuesta es viable (...) su propuesta tiene supuestos en base a los cuales se hace viable (...) con los supuestos que ellos colocaron, que además parecían conservadores, su propuesta se veía como viable, qué queri’ que te diga ${ }^{95}$.

No obstante, la Asociación de AFP - organización gremial de las administradoras de pensiones - se ha mostrado crítica a la propuesta del movimiento social, indicando que no tiene viabilidad por el cambio demográfico que se estaría dando en Chile (envejecimiento de la población) y el costo estatal.

El problema que tiene esto que a poco andar se empiezan a generar déficit, déficit que, de aquí a 30 años, o sea en el año 2050 obligaría a que el estado, para mantener el nivel de beneficios deba suplementar las pensiones de los jubilados en un 50\% y al 2100 el estado debería suplementar para aumentar los beneficios un 75\%, entonces no tiene, no tiene un sustento demográfico que permita viabilizar en el largo plazo esa propuesta (...) no va a dar en Chile, la cantidad de trabajadores activos por cada pensionado hoy día estamos cerca de cuatro aquí en Chile y el año 2050 vamos a estar en el dos y medio y el 2100 vamos a estar casi uno a uno ${ }^{96}$.

\section{Repertorio de estrategias en contexto de elecciones nacionales (2017)}

Durante las elecciones presidenciales y parlamentarias de 2017 se produce la segunda innovación en el repertorio de estrategias de No Más AFP, la realización de un plebiscito durante el 29 de septiembre y el 1 de octubre, en el que se votaba si se quería terminar o no con el sistema actual. El plebiscito fue auto-gestionado, levantado por voluntarios, y se desarrolló de forma presencial y online.

En esta actividad votaron más de un millón de personas, donde el 97\% votó por la opción de terminar con las AFP, mientras que tan solo un 1,7\% estuvo a favor de seguir con el sistema de capitalización individual ${ }^{97}$. Con $90 \%$ de las actas escrutadas, la Coordinadora cifró la votación online en 417.477 votos y la votación presencial fue de 575.998 votos $^{98}$.

Esta acción responde a la coyuntura política eleccionaria, en el que el cambio de estrategia estaba consignado para hacerle frente al marco mediático de los comicios y su preminencia en la discusión

94 Entrevista n ${ }^{\circ}$, Dirigente de No Más AFP, 18 de julio de 2018.

95 Entrevista $n^{\circ} 7$, Autoridad de Gobierno, 10 de mayo de 2018.

${ }^{96}$ Entrevista $n^{\circ}$ 14, miembro de la Asociación de AFP, 12 de julio de 2018.

97 ADN Radio, "Gobierno de Piñera ingresará su proyecto de reforma a las pensiones el primer semestre de este año". 8 de marzo, 2018, disponible: http://www.adnradio.cl/noticias/economia/gobierno-de-pinera-ingresara-su-proyecto-de-reforma-alas-pensiones-el-primer-semestre-de-este-ano/20180308/nota/3720958.aspx, revisado: 26 de mayo de 2018.

98 CONFUSAM, "Resultados Plebiscito No + AFP", CONFUSAM, 4 de octubre, 2017, disponible en: https://confusam.cl/index.php/2017/10/04/resultados-plebiscito-noafp/, revisado: 26 de mayo de 2018. 
política. "Venía un período electoral que iba a ser sumamente difícil que nos iba a tapar, digamos, que nos iba, que lo electoral se lo iba a comer todo ¿Cómo salíamos a enfrentar eso? Con el plebiscito"99.

Así mismo, el plebiscito también fue una decisión estratégica del movimiento para evitar el desgaste de las marchas y la pérdida de adhesión ciudadana con la causa.

No basta con marchar, evidentemente hemos tenido unas marchas maravillosas, como hace muchos años en Chile no lo había, y la gente está, pero no se oye [la demanda] (...) finalmente tenemos que buscar otras formas y entonces dijimos bueno, utilicemos este mecanismo que sí existe en otras, en otros países, que es un mecanismo democrático, y sigamos agitando bajo este recurso para que sigamos sumando fuerzas y sigamos activos y no caigamos en la inmovilidad ${ }^{100}$.

Este hecho muestra la importancia analítica de considerar los factores internos y externos en la innovación de los repertorios de estrategias, puesto que No Más AFP consideró el plebiscito como forma de hacer frente a la coyuntura electoral y buscar formas para mantener el apoyo social.

No obstante, esta no fue la única acción que desarrolló el movimiento durante elecciones. En el período de campaña, No Más AFP sostuvo reuniones con la mayoría de las candidaturas presidenciales. En esta línea, pasaremos a revisar la relación entre la Coordinadora y las tres principales campañas presidenciales, Sebastián Piñera (Chile Vamos), Alejandro Guillier (Fuerza de Mayoría) y Beatriz Sánchez (Frente Amplio). La estrategia de reunirse con candidaturas presidenciales muestra la relevancia de superar la dicotomía entre acciones contenciosas y rutinarias ${ }^{101}$, ya que movimientos como No Más AFP tienen repertorios en el que confluyen acciones insiders como outsiders. Este tipo de acciones corresponde a una combinación entre determinantes contextuales e internas porque es parte de una definición estratégica del movimiento y marcar presencia mediática en un escenario político intrínsecamente demarcado por las elecciones nacionales.

El programa de gobierno de Sebastián Piñera en materia de pensiones proponía como principales iniciativas aumentar en 4\% la cotización con cargo al empleador y que sea administrado por las AFP y aumentar en un $42 \%$ el pilar solidario del sistema para mejorar la pensión básica y aporte previsional solidario. De esta manera, las propuestas de Piñera buscan corregir las externalidades negativas de la capitalización individual a partir de subsidios competitivos e incentivando la competencia en la industria de AFP, cuestión propia de las políticas neoliberales.

En entrevistas, distintos dirigentes nacionales de la Coordinadora señalaron que no hubo acercamientos con la campaña de Sebastián Piñera por haber una gran distancia entre la propuesta de No Más AFP y la visión de la derecha en materia de pensiones.

Por su parte, el programa de Alejandro Guillier proponía aumentar en un 5\% la cotización con cargo al empleador y que ese porcentaje sea administrado por un ente estatal, además de dar la posibilidad de que los trabajadores puedan optar a que dicha agencia pública administre su 10\% de cotización.

Respecto de la propuesta de No + AFP, Andras Uthoff, parte del equipo programático de Guillier, ex miembro de la Comisión Bravo y parte del equipo de expertos que asesoró a la Coordinadora en la construcción de su propuesta técnica, dijo que

Adherirnos a [la propuesta de] No +AFP, en ningún caso, pero sí entender que se debe terminar con los abusos (...). Guillier es consciente de que hay un fondo de pensiones de ciertas magnitudes que es importante en los equilibrios macro; está consciente que es de propiedad de los trabajadores,

\footnotetext{
${ }^{99}$ Entrevista $n^{\circ} 1$, op. cit.

100 Entrevista $n^{\circ} 13$, op. cit.

${ }^{101}$ Donoso y von Bülow, op. cit;; Rossi, op. cit; Pettinicchio, op. cit.
} 
pero también está consciente de que los trabajadores están disconformes con las pensiones que da el sistema ${ }^{102}$.

En cuanto al programa de Beatriz Sánchez, el Frente Amplio hizo suya la propuesta de cambio al sistema de capitalización individual de No Más AFP, proponiendo un sistema de reparto solidario y tripartito. La candidata del Frente Amplio señaló que durante los primeros 100 días de gobierno ingresaría un proyecto de ley que crea un sistema nacional de seguridad social y termina con las AFP103.

Sobre la adhesión a la propuesta de No Más AFP, un miembro del equipo programático del Frente Amplio dijo lo siguiente,

Tradujimos un poco la propuesta para que sea una propuesta del Frente Amplio también, y ahí cambiamos un poquito el lenguaje, pero es básicamente la misma propuesta, y antes de eso, antes que todo, antes que Beatriz [Sánchez] incluso, fuimos generando los vínculos, porque también dentro del Frente Amplio hay militantes que son además participantes de la Coordinadora, a través de ellos nos fuimos juntando ${ }^{104}$.

A segunda vuelta pasaron Piñera y Guillier y la Coordinadora volcó su estrategia a tensionar a la candidatura del oficialismo para que expusiera su posición sobre la propuesta de cambio del sistema del movimiento. En este escenario, la Coordinadora tuvo una reunión con Guillier. Finalizada la conversación, Luis Mesina dijo que Guillier "se comprometió a transitar en esa dirección, plebiscitar si es necesario las propuestas que tiene el mundo del trabajo, es decir, ejercer la democracia, devolver la soberanía al pueblo" y agregó que "no vamos a llamar a votar por Alejandro Guillier. No llamamos a votar por Beatriz Sánchez que tomó en sus manos nuestra propuesta"105. Sin embargo, Guillier no daría una posición clara respecto a la propuesta de la Coordinadora de cara a la segunda vuelta.

Vale mencionar, que antes que el Frente Amplio definiera a Beatriz Sánchez como su candidata a la Presidencia, parte de la coalición buscó levantar a Luis Mesina como candidato a La Moneda, lo que muestra la cercanía entre el movimiento y dicho pacto político. Sin embargo, No Más AFP decidió descarta la opción de llevar a su principal dirigente a la carrera presidencial.

Sobre la reunión entre No Más AFP y el comando de Guillier, un miembro del equipo orgánico de la campaña de Guillier ${ }^{106}$ sostuvo que

el contacto con el movimiento No Más AFP no fue más que al inicio y al final con reuniones, pero no tuvimos un vínculo mayor, entre otras cosas porque ellos, mi impresión y eso no significa que sea negativo en lo absoluto, ellos estaban bien enmarcados con el Frente Amplio, y su vez el Frente Amplio había comprado la consigna y, por lo tanto, nos parecía que era, en primer lugar, oportunista sumarse y, en segundo lugar, teníamos algunas diferencias respecto de lo que había que hacer del mediano y largo plazo, de manera que no tuvimos un diálogo permanente, no tuvimos un diálogo fluido, ni tampoco lo buscamos.

Si bien el movimiento se reunió con distintas candidaturas presidenciales, no hizo un llamado a votar por ningún conglomerado. Esta decisión tiene que ver con una definición política de No Más AFP,

102 La Tercera, "Uthoff: 'Adherirnos al No+AFP, en ningún caso", 24 de noviembre, 2017b, disponible en: http://www2.latercera.com/noticia/uthoff-adherimos-al-noafp-ningun-caso/, revisado: 20 de mayo de 2018.

103 La Tercera, "Las medidas para los primeros 100 días de los candidatos a La Moneda", 17 de noviembre, 2017c, disponible en: http://www2.latercera.com/noticia/las-medidas-los-primeros-100-dias-los-candidatos-moneda/, revisado: 20 de mayo de 2018.

104 Entrevista n 6, 9 de mayo, 2018.

105 La Tercera, "Vocero de movimiento No + AFP: 'No vamos a llamar a votar por Alejandro Guillier", 30 de noviembre, 2017d, disponible en: http://www2.latercera.com/noticia/vocero-del-movimiento-noafp-no-llamar-votar-alejandro-guillier/, revisado: 20 de mayo de 2018.

106 Entrevista n 8, 15 de mayo, 2018. 
que es conservar su autonomía política respecto a los partidos políticos. "No más AFP siempre ha tratado de mantenerse como un sector organizado (...) de trabajadores o de pobladores que no están contaminados por el mundo político"107.

Este principio tiene directa relación con que los movimientos sociales en Chile cada vez dependen menos de los polity members ${ }^{108}$, lo que aplicaría para el caso de los repertorios de estrategias, desafiando a la literatura que ve una relación positiva entre la presencia de aliados en el sistema político y el desarrollo de repertorios ${ }^{109}$.

\section{El repertorio de estrategias de No Más AFP en el gobierno de Piñera}

En las elecciones presidenciales de 2017, se impuso Sebastián Piñera por 54,4\% de los votos por sobre Alejandro Guillier. Este hecho abre un nuevo escenario político para la discusión sobre cambios al sistema de pensiones. Durante el primer mes de su gobierno, Piñera convocó a un Comité Técnico para la reforma previsional. Este Comité está dirigido por Augusto Iglesias, jefe programático de la candidatura presidencial de Sebastián Piñera en 2017 y ex Subsecretario de Previsión Social, e integrado por los Ministerios de Hacienda y Trabajo, además de los asesores presidenciales encabezados por Cristián Larroulet ${ }^{110}$.

En este escenario, la innovación que está preparando el movimiento es la de la "iniciativa popular de ley". Esta acción consiste en transformar la propuesta de sistema de reparto de la Coordinadora a un proyecto de ley que pueda ser pulido y discutido por la ciudadanía en espacios locales a lo largo del país y que sea patrocinada por los parlamentarios que han apoyada la propuesta del movimiento. La Coordinadora ve en esta acción la posibilidad de capitalizar la participación en una posterior huelga general ${ }^{111}$.

(...) cuando nosotros hemos planteado la iniciativa popular de ley, lo que estamos planteando es devolver el poder soberano al pueblo y que el pueblo entonces sea quien también tenga la posibilidad de legislar o ser parte de la iniciativa legislativa, entonces, nosotros vemos esto de la iniciativa popular de ley como una forma de movilización, en que podamos (...) traducir esta propuesta en un proyecto de ley que podamos levantar con el apoyo de la gente ${ }^{112}$.

Al mismo tiempo, otro objetivo detrás de esta estrategia es abrir un nuevo proceso de concientización y educación ciudadana que tenga como repercusión dificultar el escenario para la reforma de pensiones que busca implementar el actual gobierno de Piñera. En este sentido, esta acción también está asociada a la importancia que No Más AFP ve en la autonomía política respecto a los polity members, ya que supone una acción de presión política desde la sociedad organizada y que busca influir en la toma de decisiones

Esta iniciativa popular de ley nos reinstala en la discusión en la ciudadanía para volver a generar esa necesidad de una reforma a la previsión (...) la apuesta de la coordinadora es ver cómo somos capaces de reinstalar la discusión para futuros gobiernos y me parece que la iniciativa va en esa dirección, es decir volver a decirle a la gente que el sistema es malo y que, por lo tanto, lo que hay que hacer es una reforma completa al sistema previsional, reinstalando el concepto de seguridad social como eje central, y ese va a

\footnotetext{
107 Entrevista ${ }^{\circ} 5$, op. cit.

108 Somma y Medel, op. cit.; Somma y Bargsted, op. cit.

109 Tilly y Tarrow, op. cit.; Tarrow, op. cit.; Tilly, op. cit.; McAdam, op. cit.

110 Ex Ministro Secretario General de la Presidencia durante el primer gobierno de Sebastián Piñera (2010-2014).

111 No Más AFP, "Proponemos restituir soberanía al pueblo", Coordinadora No Más AFP, 23 de abril, 2018, disponible en: https://coordinadoranomasafp.cl/wp/2018/04/23/d-i-s-c-u-r-s-o-c-e-n-t-r-a-l-marcha-noafp-22-de-abril/, revisado: 19 de mayo de 2018.

112 Entrevista ${ }^{\circ}$ 2, op. cit.
} 
ser nuestro gran desafío en este tiempo, es decir reinstalar en la ciudadanía la necesidad de un cambio al sistema previsional ${ }^{113}$.

\section{Conclusiones}

Desde la irrupción de las movilizaciones de 2016, el movimiento No Más AFP ha realizado una serie de acciones variadas e innovadoras, conformando un repertorio de estrategias amplio y diverso. Como muestra este artículo, para desarrollar un análisis profundo y minucioso de los repertorios de estrategias de los movimientos sociales es necesario dejar de diferenciar entre acciones institucionales y extrainstitucionales, ya que las acciones son dinámicas y pueden tener características tanto contenciosas como rutinarias ${ }^{114}$.

En tanto, los principales hallazgos son que las innovaciones de estrategias de No Más AFP están asociadas a coyunturas políticas particulares - caso Myriam Olate, beneficios tributarios de AFP y elecciones nacionales - como también factores internos - la centralidad simbólica de la huelga general y concientización de la importancia de la autonomía política del movimiento social. Es necesario subrayar que este estudio entrega evidencia relevante sobre el distanciamiento entre movimientos sociales y partidos políticos en Chile, al mostrar que los movimientos que conservan y definen una autonomía respecto a los partidos, desarrollando repertorios de estrategias diversas e innovadoras porque no dependen de los recursos y los intereses de los polity members.

Este artículo aporta a la literatura sobre movimientos sociales y, particularmente, a los estudios sobre repertorios de estrategias en Chile al entregar una perspectiva que va más allá de la dicotomía clásica entre acciones institucionales y extra-institucionales. Por su parte, la noción de innovación en los repertorios de estrategias es un aspecto poco estudiado en Chile y este estudio da cuenta de la relevancia del cambio en repertorio como una actividad estratégica por parte de los movimientos sociales. En este sentido, este artículo abre una línea de investigación en el análisis de la movilización social en el caso chileno. A su vez, entrega antecedentes relevantes sobre el movimiento No Más AFP, organización social clave de los ciclos de protesta post 2011 y que ha sido poco estudiado.

Finalmente, el escenario político para el movimiento No Más AFP es complejo, ya que el gobierno de Piñera tiene por objetivo introducir modificaciones al sistema actual de pensiones, aumentando la cotización en un 4\%, porcentaje que irá íntegro a las aseguradoras de fondos de pensiones. En esta línea, el repertorio estratégico de No Más AFP se encuentra abierto para afrontar el contexto político actual y sólo el resultado del policy making podrá mostrar la efectividad de la iniciativa popular de ley y otras acciones que se puedan ir fraguando a futuro por parte del movimiento social.

\section{Bibliografía}

\section{Periódicos}

ADN Radio. "Gobierno de Piñera ingresará su proyecto de reforma a las pensiones el primer semestre de este año". 8 de marzo, 2018. Disponible: http://www.adnradio.cl/noticias/economia/gobierno-de-pinera-ingresara-su-proyecto-de-reforma-alas-pensiones-el-primer-semestre-de-este-ano/20180308/nota/3720958.aspx. Revisado: 26 de mayo de 2018.

El Mercurio. "Esta es la propuesta de cambio al sistema de pensiones de No + AFP", ElMercurio, 29 de noviembre, 2016. Disponible en: http://www.elmercurio.com/Inversiones/Noticias/Fondos-de-

\footnotetext{
${ }^{113}$ Entrevista n ${ }^{\circ} 15$, op. cit.

114 Donoso y von Bülow, op. cit.; Rossi, op. cit.; Pettinicchio, op. cit.
} 
Pensiones/2016/11/29/Esta-es-la-propuesta-de-cambio-al-sistema-de-pensiones-de-NoAFP.aspx.

Revisado: 28 de mayo de 2015.

Emol. "Ex subdirectora técnica de Gendarmería por pensión de \$5 millones: 'Es la que corresponde en derecho", Emol, 05 de julio, 2016. Disponible en: http://www.emol.com/noticias/Nacional/2016/07/05/810948/Myriam-Olate-defiende-pension-de-5millones-Es-la-que-corresponde-en-derecho.html. Revisado: 14 de mayo de 2018.

La Tercera. "Coordinadora "No + AFP" calificó como histórica la jornada de movilización", 26 de marzo, 2017a. Disponible en: http://www2.latercera.com/noticia/hoy-se-realiza-una-nueva-marchaconvocada-noafp/. Revisado: 14 de mayo de 2018.

La Tercera. "Uthoff: "Adherirnos al No+AFP, en ningún caso", 24 de noviembre, 2017b.

Disponible en: http://www2.latercera.com/noticia/uthoff-adherirnos-al-noafp-ningun-caso/.

Revisado: 20 de mayo de 2018.

La Tercera. "Las medidas para los primeros 100 días de los candidatos a La Moneda". 17 de noviembre, 2017c. Disponible en: http://www2.latercera.com/noticia/las-medidas-los-primeros-100dias-los-candidatos-moneda/. Revisado: 20 de mayo de 2018.

La Tercera. "Vocero de movimiento No + AFP: 'No vamos a llamar a votar por Alejandro Guillier", 30 de noviembre, 2017d. Disponible en: http://www2.latercera.com/noticia/vocero-delmovimiento-noafp-no-llamar-votar-alejandro-guillier/. Revisado: 20 de mayo de 2018.

La Tercera. "La historia del movimiento que convocó a miles de chilenos", 26 de julio, 2016 a. Disponible en: http://www2.latercera.com/noticia/la-historia-del-movimiento-que-convoco-a-milesde-chilenos/. Revisado: 14 de mayo de 2018.

La Tercera. "No + AFP cambia de estrategia y radicaliza presión al gobierno", 4 de noviembre, 2016b. Disponible en: http://www2.latercera.com/noticia/noafp-cambia-estrategia-radicaliza-presional-gobierno/. Revisado: 14 de mayo de 2018.

La Tercera. "SII recopila antecedentes por fusión entre AFP Provida y Acquisition", 3 de marzo, 2016c. Disponible en: http://www2.latercera.com/noticia/sii-recopila-antecedentes-por-fusion-entreafp-provida-y-acquisition/. Revisado: 14 de mayo de 2018.

La Tercera. "Director del SII califica como "legal" la fusión entre Cuprum y Argentum", 6 de octubre, 2015. Disponible en: http://www2.latercera.com/noticia/director-del-sii-califica-como-legal-lafusion-entre-cuprum-y-argentum/. Revisado: 26 de mayo de 2018.

\section{Bibliografía citada}

Bidegain, Germán. "From Cooperation to Confrontation: The Mapuche Movement and Its Political Impact, 1990-2014", en Sofía Donoso y Marisa von Bülow (compiladoras), Social Movements in Chile. Organization, trajectories \& political consequences, Nueva York, Palgraves Macmillan, 2017.

Bidegain, Germán. Autonomización de los movimientos sociales e intensificación de la protesta: estudiantes y mapuches en Chile (1990-2013). Tesis Doctoral en Ciencia Política, Instituto de Ciencia Política, Pontificia Universidad Católica de Chile, 2015.

Cress, Daniel y Snow, David. "The outcomes of homeless mobilization: the influence of organization, political mediation, and framing", American Journal of Sociology 105: 4, 2000, 1063-1104.

Dalton, Russell, Van Sickle, Alix, y Weldon, Steven. "The individual-institutional nexus of protest behavior", British Journal of Political Science 40: 1, 2010, 51-73.

Delamaza, Gonzalo, Maillet, Antoine y Martínez, Christian. "Socio-territorial conflicts in Chile: configuration and politicization (2005-2014)", European Review of Latin American and Caribbean Studies 104, 2017, 23-46.

Donoso, Sofía. “'Outsider' and 'insider' strategies: Chile's student movement, 1990-2014”, en Sofía Donoso y Marisa von Bülow (compiladoras), Social Movements in Chile. Organization, trajectories \& political consequences, Nueva York, Palgraves Macmillan, 2017. 
Donoso, Sofía. "Dynamics of change in Chile: explaining the emergence of the 2006 pingüino movement", Journal of Latin American Studies 45: 1, 2013, 1-29.

Donoso, Sofía y von Bülow, Marisa. "Introduction: social movements in contemporary Chile", en Sofía Donoso y Marisa von Bülow (compiladoras), Social Movements in Chile. Organization, trajectories \& political consequences, Nueva York, Palgraves Macmillan, 2017.

Garretón, Manuel Antonio. La gran ruptura. Institucionalidad politica y actores sociales en el Chile del Siglo XXI, Santiago, LOM ediciones, 2016.

Goldstone, Jack. "More social movements or fewer? Beyond political opportunity structures to relational fields", Theory and Society 33, 2004, 333-365.

Gutierrez, Francisca. (2017). "Coping with neoliberalism through legal mobilization: the chilean labor movement's new tactics and allies", en Sofía Donoso y Marisa von Bülow (compiladoras), Social Movements in Chile. Organization, trajectories \& political consequences, Nueva York, Palgraves Macmillan, 2017.

Hanna, Phillipe, Vanclay, Frank, Langdon, Esther Jean y Arts, Jos. "Conceptualizing social protest and the significance of protest actions to large projects", The Extractive Industries and Society 3, 2016, 217-239.

Hevia de la Jara, Felipe. "La difícil articulación entre políticas universales y programas focalizados. Etnografía institucional del programa Bolsa Familia de Brasil”, Gestión y Política Pública, XX: 2, 2011, 331-379.

Jenkins, Craig. "La Teoría de la Movilización de Recursos y el Estudio de los Movimientos Sociales", Zona Abierta 69, 1994, 5-49.

Kriesi, Hanspeter. "Political Context and Opportunity", en David Snow, Sarah A. Soule y Hanspeter Kriesi (compiladores), The Blackwell Companion to Social Movements, Oxford, Blackwell Publishing, 2006.

Luna, Juan Pablo y Filgueira, Fernando. "The left turns as multiple paradigmatic crises", Third World Quarterly 30: 2, 2009, 371-395.

Maillet, Antoine. "Más allá del 'modelo' chileno: una aproximación multi-sectorial a las relaciones Estado-mercado”, Revista de Sociología e Política 23: 55, 2015, 53-73.

Manzano, Cristopher. La Asamblea de la Civilidad. Movilización social contra la dictadura en los 80. Santiago, Londres 38, 2014.

McAdam, Doug. Political Process and the Development of Black. Insurgency, 1930-1970. Chicago, University of Chicago Press, 1999, segunda edición.

McAdam, Doug, Tarrow, Sidney y Tilly, Charles. Dynamics of Contention. Cambridge, Cambridge University Press, 2001.

McCammon, Holly. The U.S. Women's Jury Movements and Strategic Adaptation: A More Just Verdict. Nueva York, Cambridge University Press, 2012.

McCarthy, John y Zald, Meyer. "Resource Mobilization and Social Movements: A Partial Theory", American Joumal of Sociology 82: 6, 1997, 1212-1241.

McCurdy, Patrick, Feigenbaum, Anna y Frenzel, Fabian. "Protest camp and repertoires of contention", Journal of Social, Cultural and Political Protest 15: 1, 2015, 97-104.

Medel, Rodrigo y Somma, Nicolás "¿Marchas, ocupaciones o barricadas? Explorando las determinantes de las tácticas de la protesta en Chile", Política y gobierno XXIII: 1, 2016, 163-199.

Mesa-Lago, Carmelo. "Reformas estructurales y re-reformas de pensiones, y sus efectos en el bienestar social: el caso de Chile", Politica y Sociedad 52: 3, 2015, 719-739.

Mesa-Lago, Carmelo y Bertranou, Felipe. "Pension reforms in Chile and social security principles, 1981-2015”, International Social Security Review 69: 1, 2016, 25-45.

Meyer, David. "Protest and political opportunities", Annual Review of Sociology 30, 2004, 125-45.

Meyer, David y Minkoff, Debra. "Conceptualizing Political Opportunity", Social Forces 82: 4, 2004, 1457-1493. 
98.

Meyer, David y Whittier, Nancy. "Social Movement Spillover”, Social Problems 41: 2, 1994, 277 -

Morris, Aldon. "Black Southern Student Sit-In Movement: An Analysis of Internal Organization", American Sociological Review 46: 6, 1981, 744-67.

Muñoz, Víctor y Durán, Carlos. "Los jóvenes, la política y los movimientos en el Chile reciente. Ciclos sociopolíticos entre 1967 y 2017”, Izquierdas 45: febrero, 2019, 129-159.

No Más AFP. "Proponemos restituir soberanía al pueblo", Coordinadora No Más AFP, 23 de abril, 2018. Disponible en: https://coordinadoranomasafp.cl/wp/2018/04/23/d-i-s-c-u-r-s-o-c-e-n-t-r-a-lmarcha-noafp-22-de-abril/. Revisado: 19 de mayo de 2018.

Pettinicchio, David. "Institutional activism: reconsidering the insider/outsider dichotomy", Sociology Compass 6: 6, 2012, 499-510.

Roberts, Kenneth. "(Re)Politicizing Inequalities: Movements, Parties, and Social Citizenship in Chile", Journal of Politics in Latin America 8: 3, 2016, 125-154.

Rossi, Federico. "Conceptualizing strategy making in a historical and collective perspective", en Federico Rossi y Marissa von Bülow (compiladores), Social movements dynamics. New perspectives on theory and research from Latin America, Londres y Nueva York: Routledge, 2015.

Ruiz, Carlos. De nuevo la sociedad. Santiago, LOM Ediciones, 2015.

Ruzza, Carlo. "Institutional actors and Italian peace movement: specializing and branching out", Theory and Society 26, 1997, 87-127.

Schaeffer, Colombina. "Democratizing the flows of democracy: Patagonia Sin Represas in the awakening of Chile's civil society", en Sofía Donoso y Marisa von Bülow (compiladoras), Social Movements in Chile. Organization, trajectories \& political consequences, Nueva York, Palgraves Macmillan, 2017.

Schussman, Alan, y Soule, Sarah. "Process and protest: Accounting for individual protest participation", Social Forces 84: 2, 2005, 1083-1108. 2000 [1990].

Scott, James. Los dominados y el arte de la resistencia. Discursos ocultos. México D. F., Ediciones Era,

Silva, Eduardo. "Post-Transition social movements in Chile in comparative perspective", en Sofía Donoso y Marisa von Bülow (compiladoras), Social Movements in Chile. Organization, trajectories \& political consequences, Nueva York, Palgraves Macmillan, 2017.

Somma, Nicolás y Medel, Rodrigo. "Shifting relationships between social movements and institutional politics", en Sofía Donoso y Marisa von Bülow (compiladoras), Social Movements in Chile. Organization, trajectories \& political consequences, Nueva York, Palgraves Macmillan, 2017.

Somma, Nicolás y Bargsted, Matías. "La autonomización de la protesta en Chile", en Juan Carlos Castillo y Cristián Cox (compiladores), Socialización política y experiencia escolar: aportes para la formación ciudadana en Chile, Santiago, Editorial del Centro de Estudios de Políticas y Prácticas en EducaciónPontificia Universidad Católica de Chile, 2015.

Spiegel, Jennifer Beth. "Performing 'in the red': transformation and tensions in repertoires of conten-tion during the 2012 Quebec student strike. Social Movement Studies", Journal of Social, Cultural and Political Protest 15: 5, 2016, 531-538.

Tarrow, Sidney. Power in Movement. Social Movements and Contencious Politics. Tercera Edición. Cambridge, Cambridge University Press, 2011, tercera edición.

Tilly, Charles. Regimes and Repertoires. Chicago, The University of Chicago Press, 2006.

Tilly, Charles y Tarrow, Sidney. Contentious politics. Nueva York, Oxford University Press, 2015, segunda edición.

Torcal, Mariano, Rodon, Toni y Hierro, María José. "Word on the street: the persistent of leftistdominated protest in Europe", West European Politics 39: 2, 2016, 326-350.

Tricot, Tokichen. "Movimiento de estudiantes en Chile: Repertorios de acción colectiva ¿algo nuevo?”, F@ro: Revista Teórica Del Departamento de Ciencias de La Comunicación, 15: 7, 2012. 
Verba, Sidney, Schlozman, Key Lehmann y Brady, Henry. Voice and Equality. Civic voluntarism in american politics. Cambridge, Massachusetts, Londres, Harvard University Press, 1995.

Von Bülow, Marisa y Bidegain, Germán. "It Takes Two to Tango: Students, Political Parties and Protest in Chile (2005-2013)" en Paul Almeida y Allen Cordero Ulate (compiladores), Handbook of Social Movements Across Latin America, Nueva York, Springer, 2015.

Wada, Takeshi. "Modularity and transferability of repertoires of contention", Social Problems 59: 4, 2012, 544-571.

Wang, Dan y Soule, Sarah. "Tactical innovation in social movements: the effects of peripherical and multi-issue protest”. American Sociological Review 81: 3, 2016, 517-548.

\section{Documentos}

Comisión Asesora para el Sistema de Pensiones. Resumen Ejecutivo. Comisión Asesora Presidencial sobre el Sistema de Pensiones, 2015. Disponible en:

http://www.comision-pensiones.cl/Documentos/Resumen. Revisado: 19 de mayo de 2018.

Confederación Bancaria. "Tras exitoso $2^{\circ}$ Congreso Nacional Movimiento No + AFP toma fuerza", Confederación de Sindicatos Bancarios y Afines, 4 de mayo, 2016. Disponible en: http://bancariachile.cl/tras-exitoso-2o-congreso-nacional-movimiento-noafp-toma-fuerza/. Revisado: 17 de mayo de 2018.

CONFUSAM. "Resultados Plebiscito No + AFP", CONFUSAM, 4 de octubre, 2017. Disponible en: https://confusam.cl/index.php/2017/10/04/resultados-plebiscito-noafp/. Revisado: 26 de mayo de 2018.

No Más AFP. Nuevo Sistema de Pensiones para Chile. Santiago, Coordinadora No Más AFP, 2016 